\title{
Size of kinematic error affects retention of locomotor adaptation in human spinal cord injury
}

\author{
Sheng-Che Yen, PT, PhD; ${ }^{1}$ Jill M. Landry, MSRS-PT; $^{2}$ Ming Wu, PhD ${ }^{2-3^{*}}$ \\ ${ }^{1}$ Department of Physical Therapy, Bouve College of Health Sciences, Northeastern University, Boston, MA; ${ }^{2}$ Sensory \\ Motor Performance Program, Rehabilitation Institute of Chicago, Chicago, IL; ${ }^{3}$ Department of Physical Medicine and \\ Rehabilitation, Feinberg School of Medicine, Northwestern University, Chicago, IL
}

\begin{abstract}
Studies in arm motor adaptation suggest that introducing small errors during the adaptation period may lead to a longer retention of the aftereffect than introducing large errors. However, it is unclear whether this notion can be generalized to locomotor adaptation in patients with incomplete spinal cord injury (SCI). We hypothesized that a smaller error size may lead to longer retention of the aftereffect in patients with SCI. We recruited 12 subjects with incomplete SCI for this study. They were instructed to walk on a treadmill while light-, medium-, and heavy-resistance loads were applied to the right ankle to perturb leg swing. Each of the three resistance-load conditions were specific to the subject and determined by each subject's maximum voluntary contraction of the hip flexors. We observed that subjects tended to make larger errors when the resistance-load condition was greater. Following resistance load release, subjects showed an aftereffect consisting of an increase in stride length. Further, the aftereffect was retained longer in the medium-resistance load condition than in the heavy- and light-resistance load conditions. This finding suggests that a patient-specific resistance load may be needed to facilitate retention of locomotor adaptation in patients with incomplete SCI.
\end{abstract}

Key words: aftereffect, error size, locomotion, motor adaptation, motor learning, resistance load, retention, spinal cord injury, stride length, treadmill.

\section{INTRODUCTION}

Motor adaptation is an error correction process that leads to recalibration of the motor command [1]. For example, when experiencing a force perturbation resisting leg flexion during the swing phase of gait, subjects initially made larger errors in leg kinematics (e.g., decreased knee flexion) [2]. The error size gradually decreased over time as the central nervous system (CNS) adapted to the force perturbation. Upon removal of the perturbation, movement aftereffects were observed in the direction opposite of the force perturbation (e.g.,

\footnotetext{
Abbreviations: $3 \mathrm{D}=$ three-dimensional, $\mathrm{C}=$ cervical, $\mathrm{CI}=$ confidence interval, $\mathrm{CNS}=$ central nervous system, $\mathrm{EMG}=$ electromyography, $\mathrm{GC}=$ gait cycle, $\mathrm{GEE}=$ generalized estimating equation, IEMG = integrated electromyography, LMM = linear mixed model, $\mathrm{MG}=$ medial gastrocnemius, $\mathrm{MVC}=$ maximum voluntary isometric contraction, $\mathrm{RF}=$ rectus femoris, $\mathrm{SCI}=$ spinal cord injury, $\mathrm{SM}=$ semitendinosus, $\mathrm{SO}=$ soleus, $\mathrm{T}=$ thoracic, $\mathrm{TA}=$ tibialis anterior, $\mathrm{VM}=$ vastus medialis. *Address all correspondence to Ming Wu, PhD; Sensory Motor Performance Program, Rehabilitation Institute of Chicago, 345 East Superior St, Rm 1406, Chicago, IL 60611; 312-238-0700; fax: 312-238-2208.

Email: w-ming@northwestern.edu

http://dx.doi.org/10.1682/JRRD.2012.09.0175
} 
increased knee flexion). The presence of the aftereffect suggests that the motor command had been updated in anticipation of the upcoming force perturbation [2-5]. These aftereffects, however, are generally short-lived. For example, aftereffects following short-term locomotor adaptation to force perturbation washed out five steps after the perturbation was removed [3].

The error size may affect the retention of aftereffects. For instance, previous arm reaching studies demonstrated that subjects can retain aftereffects longer when the perturbation was introduced in a gradual, rather than abrupt, manner during the adaptation period [6-9]. Subjects tended to make smaller errors when experiencing a gradual perturbation, while they tended to make larger errors when experiencing an abrupt perturbation [7]. It has been suggested that the neural basis for the adaptation process from large and small errors is likely different. Smaller errors produce a motor memory that has a slower rate of decay, i.e., longer retention of aftereffects. However, it is unclear whether the relationship between the error size and retention of aftereffects observed in arm motor adaptation can be generalized to locomotor adaptation. Previous studies suggest that the cerebellum is a critical structure for predictive feedforward adaptations of arm movement [10-11]. Recent studies suggest that the cerebellum is also a necessary component for locomotor adaptation [12]. We assumed that a similar mechanism may be applied in both arm and locomotor adaptations given that the cerebellum is involved in both, although locomotion involves a greater extent of spinal control through the central pattern generator [13] than arm movement.

Identifying the relationship between error size and retention of aftereffects in locomotor adaptation may have crucial clinical implications in the rehabilitation of humans with spinal cord injury (SCI). Patients with an incomplete SCI often walk with a decreased stride length and slower walking speed [14]. Previous studies have indicated that patients with incomplete SCI can adapt to a robotic-generated resistance load during gait and generate an aftereffect consisting of an increased stride length following resistance load release [15-16]. Most importantly, the aftereffect can transfer to real-life overground walking and result in an increase in walking speed [16]. However, this aftereffect is usually short-lived, limiting its clinical application in improving walking function in humans with SCI. Thus, it is crucial to determine adaptation parameters that could prolong the retention of the aftereffects in order to optimize training effect and have significant clinical implications (i.e., transfer to overground walking and improve gait patterns and speed). Previous arm adaptation studies indicated that error size may be an important parameter for modulating the retention of aftereffects [6-9]. We postulated that the error size may also modulate the retention of aftereffects in locomotor adaptation.

In this study, we tested whether retention of aftereffects could be affected by the error size introduced during locomotor adaptation to a resistance load applied to the leg during the swing phase of gait. We manipulated the error size by adjusting the amount of the resistance load and expected that the error would become larger when the resistance load became greater. In a previous study, the higher the level of resistance applied during the swing phase of gait, the greater the increase in stride length after the resistance load was removed [15], suggesting that a positive relationship exists between the error size and the magnitude of the aftereffect. However, it remains unclear how the error size affects the retention of the aftereffect during locomotor training. Here, we hypothesized that a smaller error size may lead to increased retention time of aftereffects following a short-term locomotor adaptation period in patients with incomplete SCI.

\section{METHODS}

\section{Subjects}

Twelve subjects with traumatic motor incomplete SCI were recruited for this study (Table 1). All subjects were classified as level D on the American Spinal Injury Association Impairment Scale, with SCI levels ranging from cervical (C) 1 to thoracic (T) 7. They were able to walk overground with assistive devices (e.g., cane, walker, or forearm crutches) as needed. No subjects required the use of an orthotic during daily activity or during the experiment. Inclusion criteria included subjects between 18 and $65 \mathrm{yr}$ old, medically stable with medical clearance to participate, and level of SCI between $\mathrm{C} 1$ and T10. Exclusion criteria included multiple CNS lesion sites, urinary tract infection, other secondary infections, heterotopic ossification, respiratory insufficiency, significant osteoporosis, or inability to give informed consent. 
Table 1.

Subject information.

\begin{tabular}{|c|c|c|c|c|c|c|}
\hline Subject & $\begin{array}{l}\text { Age } \\
\text { (yr) }\end{array}$ & $\begin{array}{l}\text { Time Since } \\
\text { Injury (yr) }\end{array}$ & $\begin{array}{l}\text { ASIA } \\
\text { Level }\end{array}$ & $\begin{array}{l}\text { Level of } \\
\text { Injury }\end{array}$ & $\begin{array}{l}\text { MVC } \\
(\mathrm{N})\end{array}$ & $\begin{array}{l}\text { Use of Assistive Device } \\
\text { During Daily Walking }\end{array}$ \\
\hline 1 & 48 & 3 & $\mathrm{D}$ & T5-T7 & 153.06 & Walker \\
\hline 2 & 50 & 7 & D & C6 & 125.14 & Walker \\
\hline 3 & 34 & 3 & D & C6-C7 & 81.86 & No \\
\hline 4 & 43 & 2 & D & C5-C6 & 199.83 & No \\
\hline 5 & 52 & 7 & $\mathrm{D}$ & $\mathrm{T} 7$ & 211.69 & No \\
\hline 6 & 54 & 9 & $\mathrm{D}$ & $\mathrm{C} 1-\mathrm{C} 6$ & 161.44 & No \\
\hline 7 & 41 & 6 & $\mathrm{D}$ & $\mathrm{C} 4-\mathrm{C} 5$ & 203.32 & No \\
\hline 8 & 43 & 3 & $\mathrm{D}$ & $\mathrm{C} 2-\mathrm{C} 3$ & 92.33 & Forearm Crutches \\
\hline 9 & 48 & 2 & $\mathrm{D}$ & $\mathrm{C} 6-\mathrm{C} 7$ & 96.52 & Walker \\
\hline 10 & 46 & 14 & $\mathrm{D}$ & $\mathrm{C} 5-\mathrm{C} 7$ & 190.75 & No \\
\hline 11 & 64 & 4 & $\mathrm{D}$ & $\mathrm{C} 4$ & 61.62 & Forearm Crutches \\
\hline 12 & 47 & 26 & $\mathrm{D}$ & $\mathrm{C} 5-\mathrm{C} 6$ & 107.69 & Cane \\
\hline
\end{tabular}

\section{Instrumentation}

A custom-designed, cable-driven robotic system was used to provide a controlled resistance load during the swing phase of gait (Figure 1(a)). A detailed description of the system has been reported previously [17]. In brief, the robot consists of four nylon-coated stainless-steel cables driven by motors and cable spools. The cables were designed to be attached to subjects' legs during treadmill training to provide different directions of force perturbation. In the current study, one of the cables was attached to the subject's right ankle to provide swingphase resistance (Figure 1(b)).

A customized three-dimensional (3D) position sensor was used to measure the ankle trajectory during treadmill walking (Figure 1(b)). The configuration of the sensor has been described in detail elsewhere [16]. A customized LabVIEW program (National Instruments Corporation; Austin, Texas) was used to acquire the ankle position data as well as to output the load command signals to the servomotor systems at a frequency of $1,000 \mathrm{~Hz}$.

\section{Procedures}

Each subject participated in one data collection session, which lasted about $2.5 \mathrm{~h}$. Prior to initiating the treadmill walking test, we recorded each subject's maximum voluntary isometric contraction (MVC) generated during hip flexion in the standing position (with the knee actively held in full extension) for the right leg. An instrumented cable was attached to the subject's right ankle through a strap to restrict hip flexion. The other end of the cable was attached to a frame, which was located at the back end of the treadmill, through a compression and tension load cell (MLP-100, Transducer Techniques Inc; Temecula, California). Table 1 reports the MVCs of each subject averaged across three trials. Each subject's self-selected comfortable walking speed on a treadmill without a resistance load (Table 2) was also determined and used as the test speed throughout the experiment.

The data collection session consisted of three resistance load conditions: light, medium, and heavy. Each subject completed the three conditions in a randomized order. A 10 min sitting break was inserted between test conditions. We defined the medium-resistance load as approximately 18 percent of each subject's MVC. The amount was reduced if the subject felt that he or she could not comfortably walk with the load for $10 \mathrm{~min}$. The light- and heavy-resistance loads were defined as 30 percent below and above the medium-resistance load, respectively. We did not use a heavy-resistance load more than 30 percent greater than the medium-resistance load to make sure all subjects could finish the three test conditions without significant fatigue. Table 2 reports the resistance-load conditions for each subject.

In this study, the resistance load was applied approximately from the late stance phase through the midswing phase of gait. The timing of the force application was determined based on each subject's average stance and swing times, which were determined prior to data collection for each subject. The loading was triggered by ankle position sensors based on the preset threshold. The gait events were estimated based on the ankle trajectory 
(a)

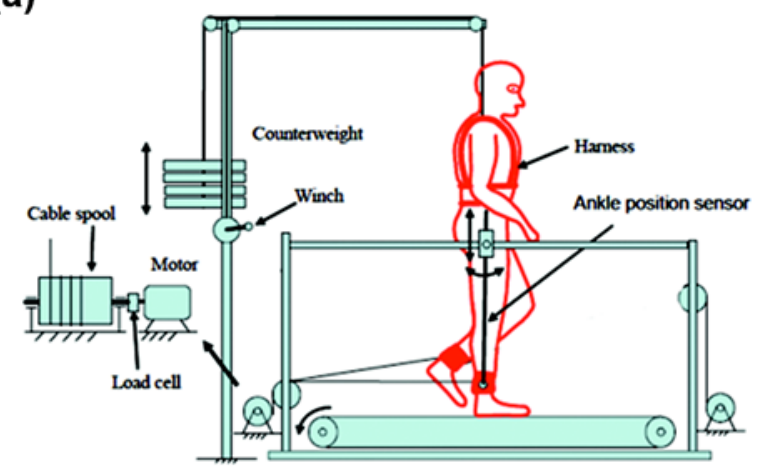

(b)

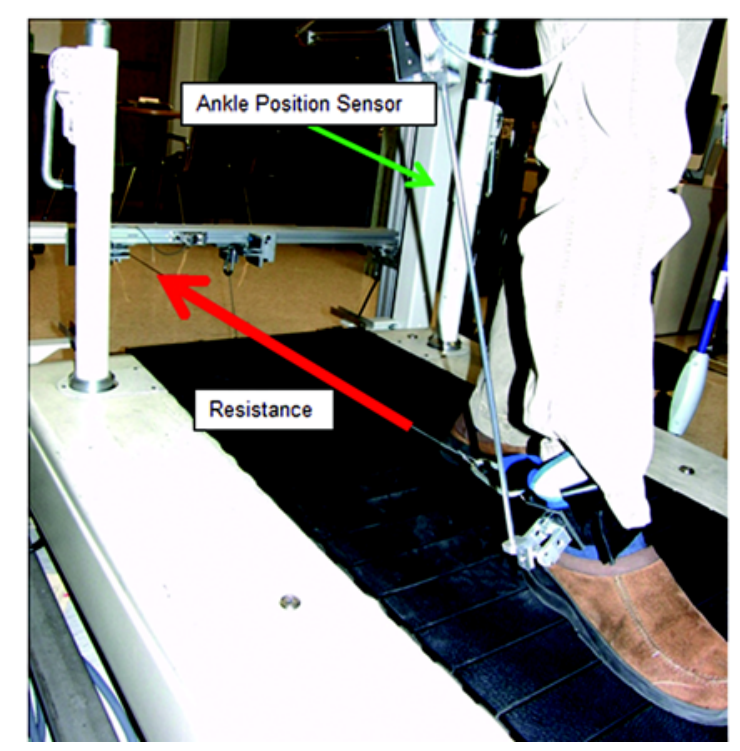

Figure 1.

(a) Cable-driven robotic system. (b) Application of resistance load at ankle.

recorded by the 3D position sensor. Specifically, heel contact was defined as the point at which the ankle movement direction changed from forward to backward; toeoff was defined as the point at which the ankle movement direction changed from backward to forward [18]. Stance phase started at heel contact and ended at toe-off, and swing phase started at toe-off and ended at the next heel contact.

Each test condition consisted of three test periods, including baseline $(1 \mathrm{~min})$, adaptation period $(7 \mathrm{~min})$, and postadaptation period $(2 \mathrm{~min})$. At baseline, subjects walked on the treadmill without resistance. In the adaptation period, subjects walked on the treadmill with the light-, medium-, or heavy-resistance loads applied to the right ankle. In the postadaptation period, subjects continued walking on the treadmill while the resistance load was released without their awareness. Each subject went through the baseline, adaptation period, and postadaptation period continuously without having a break. An overhead harness provided body-weight support as necessary to prevent knee buckling or toe dragging (Table 2). Subjects were permitted to hold on to the handrails.

The electromyography (EMG) activity of the muscles of the right leg, including the tibialis anterior (TA), medial gastrocnemius (MG), soleus (SO), vastus medialis $(\mathrm{VM})$, rectus femoris (RF), and semitendinosus (SM), were also recorded. $\mathrm{Ag}-\mathrm{AgCl}$ surface electrodes (ConMed; Utica, New York) were applied with a $2.5 \mathrm{~cm}$ center-to-center spacing over the belly of each muscle on lightly abraded skin. Active preamplifiers with shielded leads were attached to the electrodes and connected to an Octopus AMT-8 EMG unit (Bortec Biomedical Ltd; Calgary, Canada). All channels were amplified (gain $=500$ ) and sampled $(1,000 \mathrm{~Hz})$ using the same computer.

\section{Data Reduction}

The primary measure in this study was stride length. We defined stride length during treadmill walking as the horizontal distance from toe-off to the following heel contact of the same leg (i.e., the distance of leg swing). We focused on this parameter because the robotic resistance was set to affect the leg kinematics during the swing phase, and the change in swing distance can directly reflect the effect of the resistance. In addition, swing time was calculated and normalized to gait cycle (GC). Furthermore, the surface EMG signal from each muscle was low-pass filtered at $250 \mathrm{~Hz}$, high-pass filtered at $10 \mathrm{~Hz}$, and notch filtered at $60 \mathrm{~Hz}$ using a second-order Butterworth filter before rectification. The EMG data were then integrated (integrated EMG [IEMG] data) from the last 10 percent of the stance phase to the first 30 percent of swing phase (approximate to where the force was applied).

\section{Data Analysis and Statistical Analysis}

The error size of each variable measured (stride length, swing time, and IEMG) was calculated as the difference between the magnitude of each variable during the adaptation period and the magnitude during baseline. A positive error indicated that the magnitude during the adaptation period was greater than that during baseline. In contrast, a negative error indicated that the magnitude 
Table 2.

Testing parameters indicating body weight support (BWS), test speed, and resistance forces applied to leg at ankle.

\begin{tabular}{|c|c|c|c|c|c|}
\hline \multirow{2}{*}{ Subject } & \multirow{2}{*}{ BWS (\%) } & \multirow{2}{*}{$\begin{array}{c}\text { Test Speed } \\
(\mathrm{m} / \mathrm{s})\end{array}$} & \multicolumn{3}{|c|}{ Load (N) } \\
\hline & & & Light & Medium & Heavy \\
\hline 1 & 20 & 0.72 & 14 & 20 & 26 \\
\hline 2 & 0 & 0.71 & 14 & 20 & 26 \\
\hline 3 & 20 & 0.59 & 17 & 24 & 31 \\
\hline 4 & 0 & 0.82 & 22 & 31 & 40 \\
\hline 5 & 0 & 0.75 & 24 & 34 & 44 \\
\hline 6 & 0 & 0.76 & 15 & 21 & 27 \\
\hline 7 & 0 & 0.74 & 20 & 28 & 36 \\
\hline 8 & 0 & 0.47 & 8 & 11 & 14 \\
\hline 9 & 0 & 0.54 & 15 & 21 & 27 \\
\hline 10 & 0 & 0.53 & 20 & 28 & 36 \\
\hline 11 & 0 & 0.60 & 11 & 15 & 20 \\
\hline 12 & 0 & 0.50 & 17 & 24 & 31 \\
\hline
\end{tabular}

during the adaptation or postadaptation periods was smaller than during baseline. Baseline was defined as each variable's mean magnitude across the last 20 strides of baseline.

A linear mixed model (LMM) for repeated measures was used to compare the error size during the first 10 strides of the adaptation period across the three resistance load conditions. We focused on the first 10 strides of the adaptation period because previous studies suggested that error could be corrected or minimized later in this period [2-4]. The stride number (1-10) was treated as a covariate. The covariance structure between repeated measures (i.e., the loads and the strides) was modeled as compound symmetry. The LMM was also used to determine whether any significant difference existed in the baseline of each outcome measure across all resistance load conditions. Post hoc tests with the Bonferroni adjustment were conducted when a significant fixed effect of load was indicated.

To determine whether a particular resistance load condition induced an aftereffect, we compared the magnitude of the first stride during the postadaptation period and the baseline of each variable measured. Paired $t$-tests were used to determine whether the aftereffect was significant (i.e., whether the magnitude during the postadaptation period was significantly different from baseline). Due to multiple comparisons (three comparisons, one for each resistance load condition), the $p$-values were Bonferroniadjusted.

For the variable that showed a significant aftereffect, we further examined whether the retention of the aftereffect varied as a function of the load. We focused our examination on the first 30 strides of the postadaptation period. We assumed that the aftereffect was washed out if the outcome measure re-entered the 95 percent confidence interval $(\mathrm{CI})$ of the baseline value $\left(\mathrm{CI}_{\text {baseline }}\right)$ for three consecutive strides. The $\mathrm{CI}_{\text {baseline }}$ was calculated based on the subject's performance during the last 20 strides of baseline. To quantify the retention time, we counted the number of strides until the outcome measure re-entered the $\mathrm{CI}_{\text {baseline }}$ for three consecutive strides. We compared the count across the resistance load conditions using the generalized estimating equation (GEE) with Poisson loglinear model. The working correlation matrix between repeated measures (i.e., the loads) was modeled as exchangeable. Post hoc tests with the Bonferroni adjustment were conducted when a significant fixed effect of load was indicated.

All statistical analyses were conducted using Predictive Analytics Software version 18 (IBM Corporation; Armonk, New York). The $\alpha$ level for all analyses was set at $p=0.05$.

\section{RESULTS}

\section{Baseline Values of Spatial and Temporal Gait Parameters: Loaded Leg}

The baseline values of stride length, stance time, and swing time were not significantly different across the three test conditions. Specifically, the stride length was, in mean \pm standard deviation, $55.22 \pm 5.26,54.43 \pm 5.46$, and $54.98 \pm 5.57 \mathrm{~cm}$ in the light-, medium-, and heavyresistance load conditions, respectively ( $p=0.42, \mathrm{LMM})$. The swing time was $33.24 \pm 2.65,33.57 \pm 2.28$, and 
$33.95 \pm 2.96$ percent of the GC in the light-, medium-, and heavy-resistance load conditions, respectively ( $p=$ 0.16, LMM).

\section{Error Size in Spatial and Temporal Gait Parameters: Loaded Leg}

As the resistance load increased, the error size in stride length of the loaded leg increased during the first 10 strides of the adaptation period (Figure 2(a)). On average, the stride length was reduced from the baseline value by $0.65 \pm 2.83 \mathrm{~cm}$ in the medium-resistance load condition and reduced by $0.94 \pm 2.94 \mathrm{~cm}$ in the heavyresistance load condition, although slightly increased by $0.05 \pm 2.7 \mathrm{~cm}$ in the light-resistance load condition. The LMM detected a significant load effect on the error size in stride length ( $p=0.01)$, controlling for the stride number. The post hoc tests with Bonferroni correction suggested that the error size in regards to stride length was significantly greater in the heavy-resistance load condition than in the light-resistance load condition $(p=0.01)$, although no significant differences were found between the light- and medium-resistance load conditions ( $p=$ 0.14 ) and between the medium- and heavy-resistance load conditions $(p=1.00)$.

As the resistance load increased, the error size in swing time of the loaded leg increased during the first 10 strides of the adaptation period (Figure 2(b)). On average, the swing time increased from the baseline value by $0.76 \pm 2.01,1.51 \pm 2.49$, and $1.85 \pm 2.84$ percent of the GC in the light-, medium-, and heavy-resistance load conditions, respectively. The LMM detected a significant fixed effect of load on the error size of swing time $(p<$ 0.001), controlling for the stride numbers. Post hoc tests with Bonferroni correction indicated that the increase in swing time was significantly smaller in the light-resistance load condition than in the medium- $(p=0.01)$ and heavyresistance $(p<0.001)$ load conditions, although the difference between the medium- and heavy-resistance load conditions did not reach statistical significance $(p=0.49)$.

\section{Aftereffect in Spatial and Temporal Gait Parameters During First Stride of Postadaptation Period: Loaded Leg}

The resistance load induced a significant aftereffect consisting of an increase in the stride length of the loaded leg in all three conditions (Figure 3). Compared with baseline, the stride length observed during the first stride of the postadaptation period significantly increased in the (a)

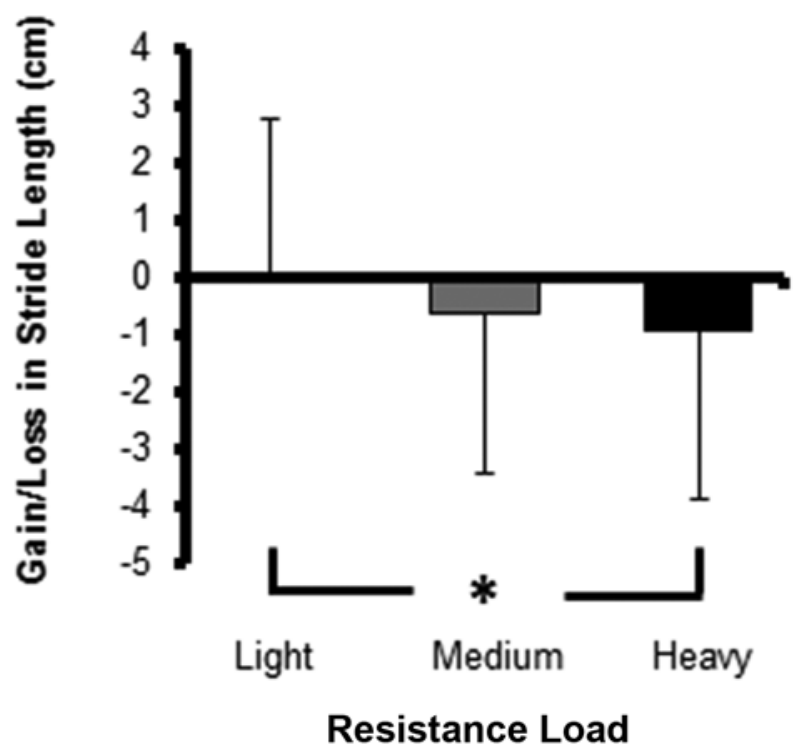

(b)

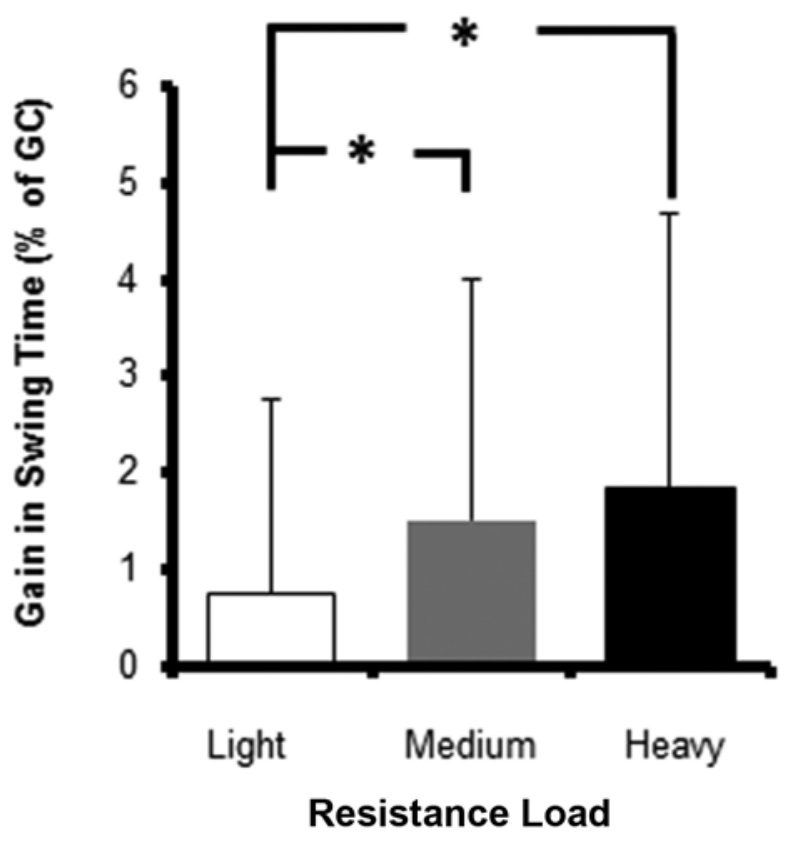

Figure 2.

Error size: mean gain/loss (across strides and subjects) in (a) stride length and (b) swing time during first 10 strides of adaptation period in each condition. Error bars represent standard deviation. ${ }^{*} p<0.05$. GC = gait cycle.

light- (baseline $=55.22 \pm 5.26 \mathrm{~cm}$ and postadaptation period $=58.43 \pm 5.30 \mathrm{~cm}$, paired $t$-tests, Bonferroni corrected $p=0.02$ ), medium- (baseline $=54.43 \pm 5.46 \mathrm{~cm}$ 


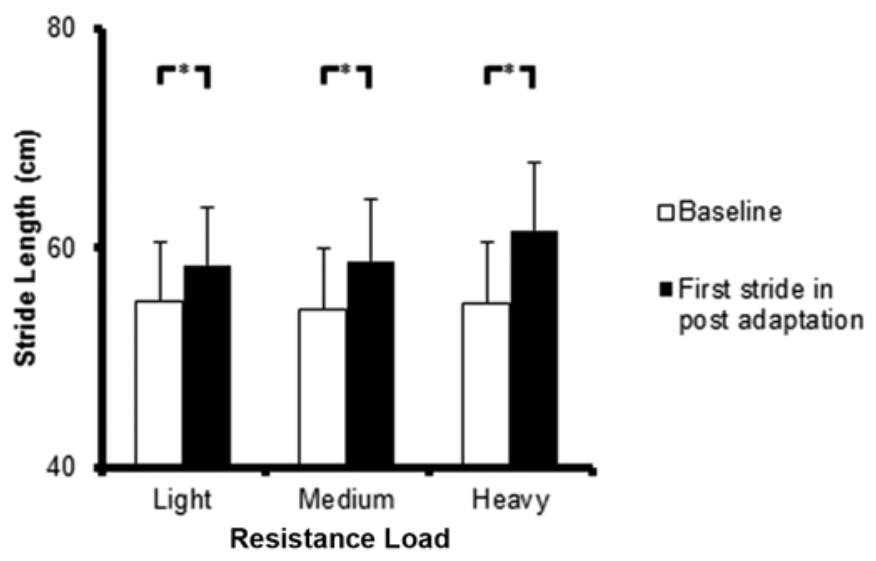

Figure 3.

Aftereffect in stride length: comparison between baseline stride length and stride length observed at first stride of postadaptation period in each condition. Error bars represent standard deviation. ${ }^{*} p<0.05$.

and postadaptation period $=58.81 \pm 5.70 \mathrm{~cm}$, Bonferroni corrected $p=0.01$ ), and heavy-resistance (baseline $=$ $54.98 \pm 5.57 \mathrm{~cm}$ and postadaptation period $=61.58 \pm$ $6.22 \mathrm{~cm}$, Bonferroni corrected $p=0.01$ ) load conditions.

The resistance load did not induce a significant aftereffect in the swing time of the loaded leg. Compared with baseline, the swing time observed during the first stride of the postadaptation period increased slightly in the light(baseline $=33.24 \% \pm 2.65 \%$ and postadaptation period $=$ $34.23 \% \pm 4.54 \%$ of GC), medium- (baseline $=33.57 \% \pm$ $2.28 \%$ and postadaptation period $=34.43 \% \pm 3.45 \%$ of GC), and heavy-resistance (baseline $=33.95 \% \pm 2.96 \%$ and postadaptation period $=35.20 \% \pm 4.61 \%$ of GC) load conditions. However, none of these changes reached statistical significance (Bonferroni corrected $p>0.6$ ).

\section{Retention of Aftereffect in Regards to Stride Length: Loaded Leg}

Retention of the aftereffect was further analyzed with regards to the stride length of the loaded leg. We did not analyze the swing time because the resistance load did not induce a significant aftereffect in this temporal variable.

Figure 4 shows the stride length from one subject during the first 30 strides of the postadaptation period and the $\mathrm{CI}_{\text {baseline }}$ for three test conditions. This subject showed an aftereffect consisting of an increased stride length at the first stride of the postadaptation period in all conditions. However, the retention of the aftereffect was different for the three load conditions, with a longer

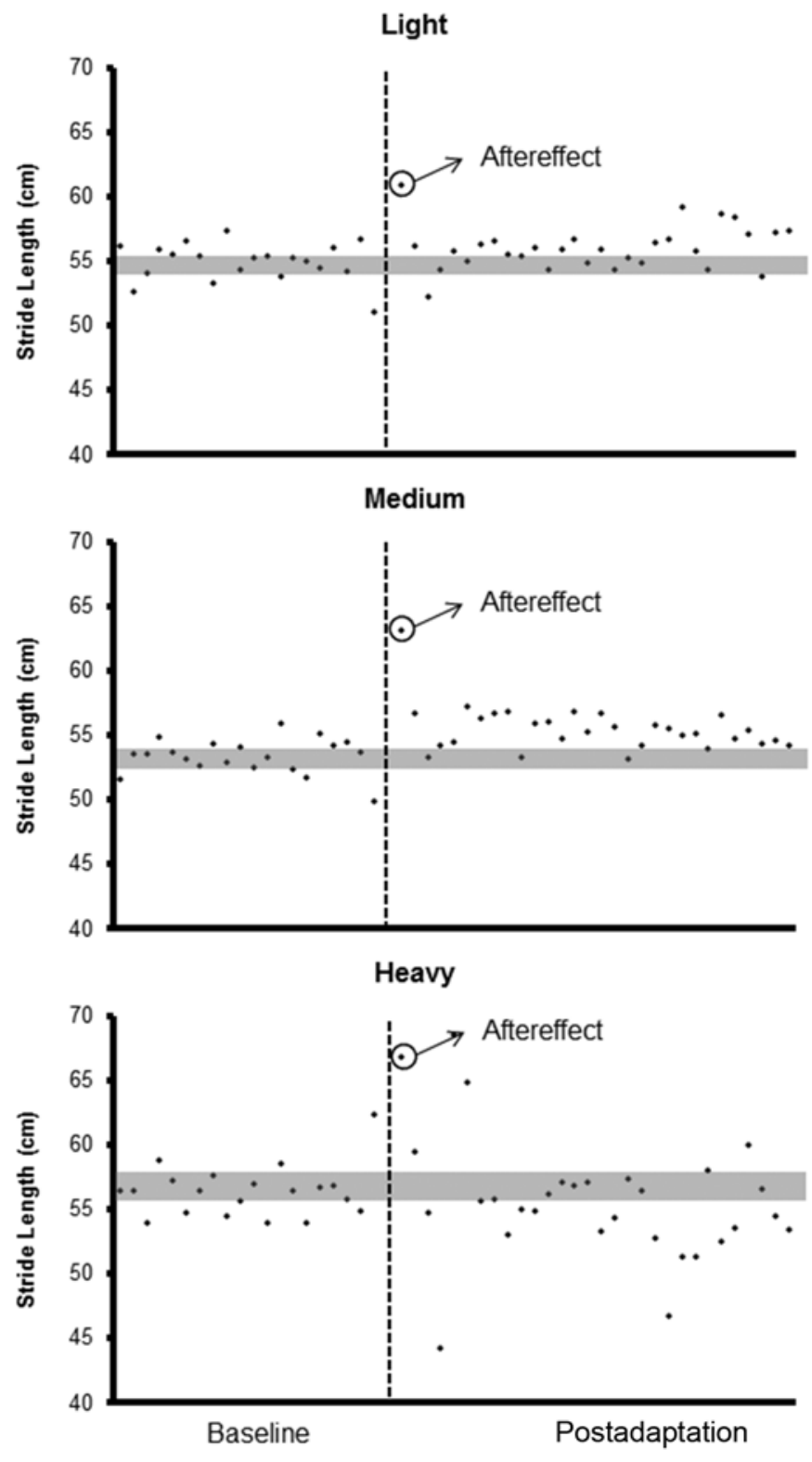

Figure 4.

Subject's stride length during last 20 strides of baseline period and first 30 strides of postadaptation period in all resistance load conditions. Shaded areas represent $\mathrm{Cl}_{\text {baseline }} \cdot \mathrm{Cl}=$ confidence interval.

retention time in the medium-resistance load condition than of that in the light- and heavy-resistance load conditions. Specifically, the subject's stride length did not fall within the $\mathrm{CI}_{\text {baseline }}$ for 3 consecutive strides during the first 30 strides of the postadaptation period in the mediumresistance load condition. In contrast, the subject's stride length fell within the $\mathrm{CI}_{\text {baseline }}$ for 3 consecutive strides 
after stride 16 in the light-resistance load condition and after stride 6 in the heavy-resistance load condition.

The group data also showed that the aftereffect in regards to stride length of the loaded leg was retained longer in the medium-resistance load condition than of that in the other two resistance load conditions (Figure 5). The GEE indicated a significant load effect on the retention of aftereffect in regards to stride length $(p=0.01)$. Post hoc tests with Bonferroni correction indicated the aftereffect was retained significantly longer in the medium-resistance load condition $(17.25 \pm 12.63$ strides $)$ than of that in the light- $(11.33 \pm 11.99$ strides, $p<0.001)$ and heavy-resistance (13.75 \pm 13.07 strides, $p=0.03)$ load conditions. There were no significant differences between the light- and heavy-resistance load conditions $(p=0.12)$.

\section{Responses of Spatial and Temporal Gait Parameters During Adaptation and Postadaptation Periods: Unloaded Leg}

No significant results were found in the spatial and temporal gait parameters of the unloaded leg during both adaptation and postadaptation periods. Although the resistance load tended to lead to an increase in stride length and a decrease in swing time in comparison to baseline during the first 10 strides of the adaptation period, none of these changes was significantly different

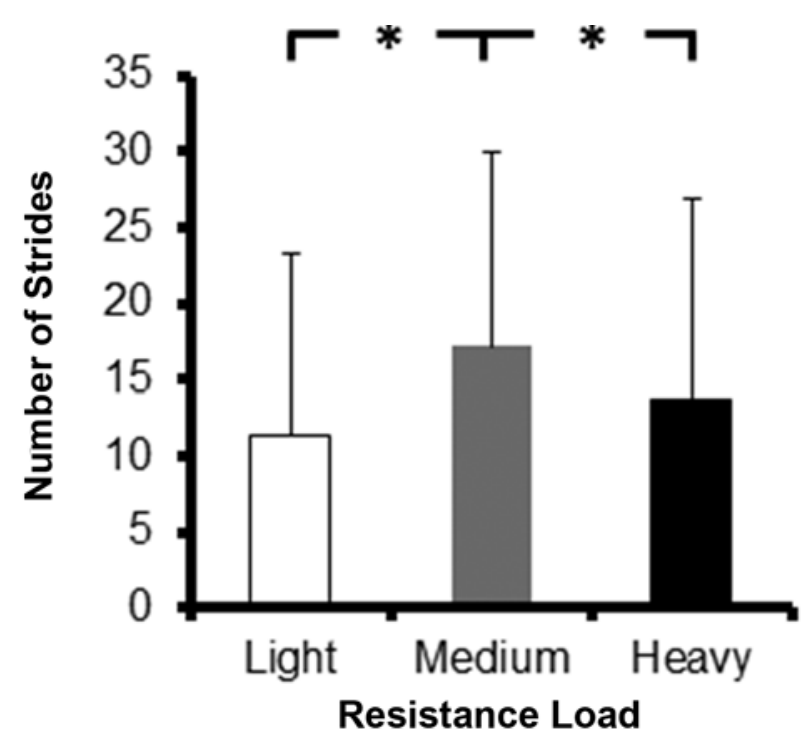

Figure 5.

Retention of aftereffect based on group. Mean number of strides before falling within or below $\mathrm{Cl}_{\text {baseline. Error bars repre- }}$ sent standard deviation. ${ }^{*} p<0.05 . \mathrm{Cl}=$ confidence interval. from one resistance load condition to another $(p>0.15$, LMM). During the first stride of the postadaptation period, the stride length increased slightly from baseline in each test condition (by approximately $1 \mathrm{~cm}$, based on the group means), but none of these changes reached statistical significance $(p>0.12)$. In addition, the swing time was slightly decreased from baseline (by $>1 \%$ of GC) in the light- and heavy-resistance load conditions, but was slightly increased from baseline (by $0.7 \%$ of GC) in the medium-resistance load condition. Again, none of these changes reached statistical significance $(p>0.45)$.

\section{Electromyography Activity During Adaptation and Postadaptation Periods}

Figure 6 shows a subject's average TA EMG (linearenveloped). During the adaptation period, the magnitude of TA EMG increased during the early swing phase of gait when the resistance load was applied for all three conditions. The amount of increase was greater when the resistance load was heavier. The mean IEMG of the group indicated a greater increase with a heavier resistance load (Figure 7). The LMM indicated that the TA IEMG varied as a function of load $(p<0.001)$. Post hoc tests with the Bonferroni correction indicated that the increase in TA IEMG was significantly smaller in the light-resistance load condition than those in the medium- $(p=0.01)$ and heavyresistance $(p<0.001)$ load conditions, although the differences between the medium- and heavy-resistance load conditions did not reach statistical significance $(p=0.45)$. The TA IEMG data from two subjects were excluded from the analysis due to incomplete recording.

Figure 8 shows a subject's average RF EMG. During the adaptation period, the magnitude of the EMG increased during the early swing phase of gait for the conditions with medium- and heavy-resistance loads, but had modest changes for the condition with the lightresistance load. The group's data indicated a greater increase in RF IEMG for the condition with mediumresistance load than that with light- and heavy-resistance load conditions (Figure 9), although the LMM indicated that those differences were not significant $(p=0.08)$. The RF IEMG data from one subject was excluded from the analysis of RF due to incomplete recording.

In addition, no significant results were found in the SO, MG, VM, and SM IEMGs during the first 10 strides of the adaptation period. In addition, paired $t$-tests indicated that there were no significant changes between 

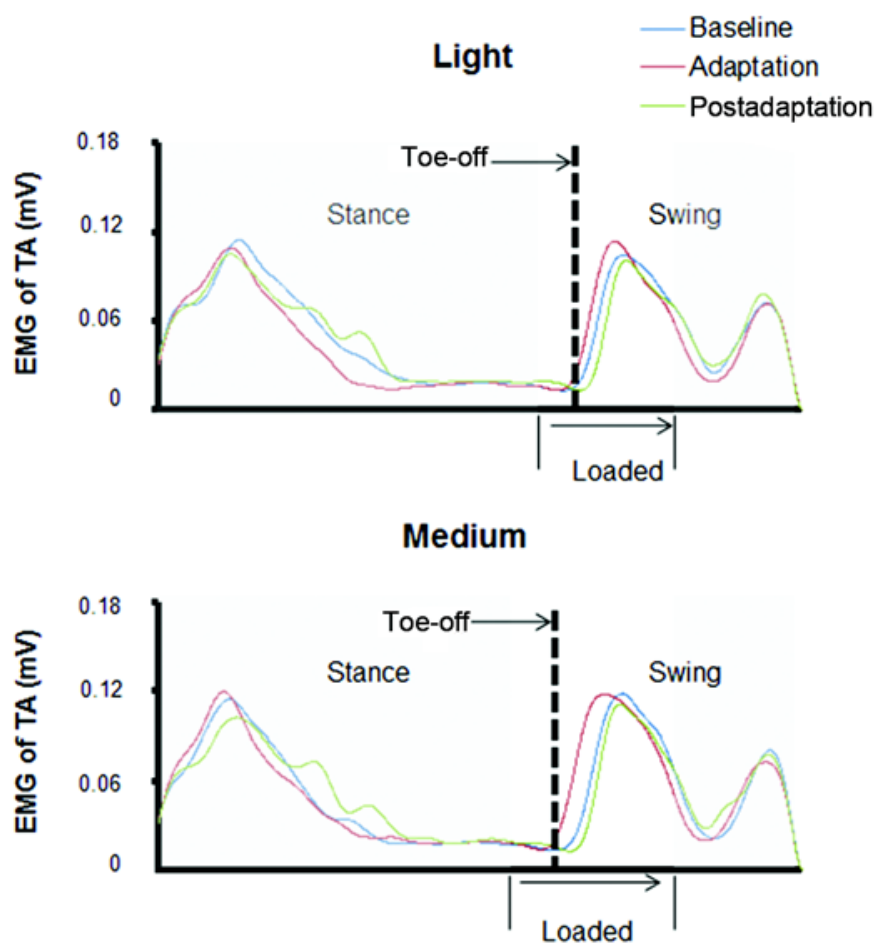

Heavy

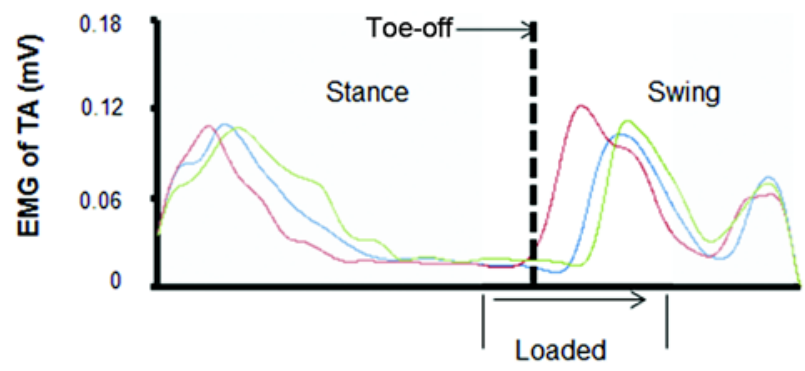

Figure 6.

Subject's tibialis anterior (TA) electromyography (EMG) pattern during baseline, adaptation period, and postadaptation period in each resistance load condition. Vertical dashed line represents event of toe-off of right leg, which separates stance and swing phases.

baseline and the first stride of the postadaptation period for all muscles $(p>0.05)$.

\section{DISCUSSION}

Our results indicated that an increase in the resistance load tended to cause an increase in error size in spatial and temporal gait parameters in human SCI. While both

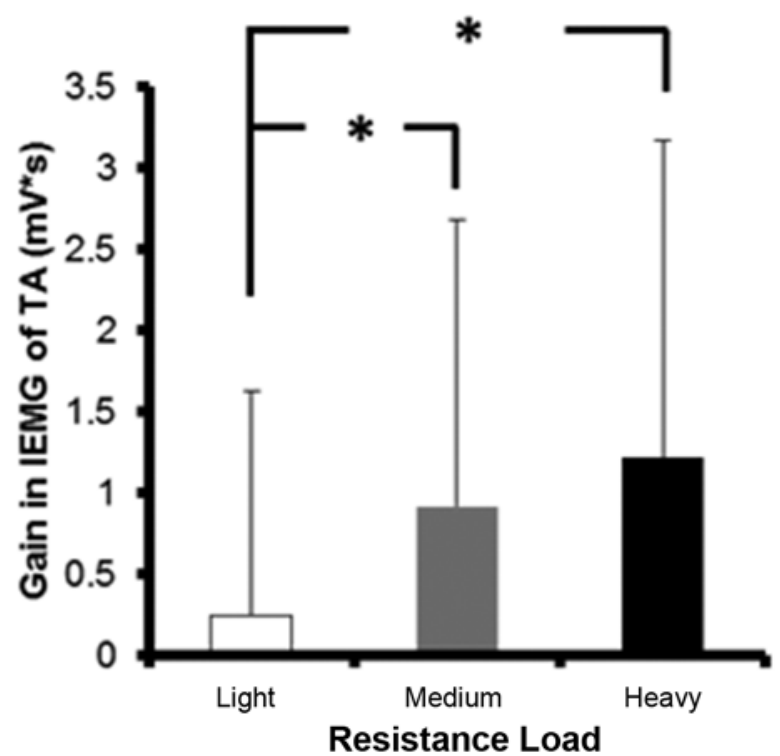

Figure 7.

Group's mean gain/loss in integrated electromyography (IEMG) of tibialis anterior (TA) during first 10 strides of adaptation period in each condition. Error bars represent standard deviation. ${ }^{*} p<0.05$.

spatial and temporal gait parameters adapted to the resistance load during the adaptation period, only the spatial gait parameter, i.e., stride length, demonstrated a significant aftereffect following resistance load release during the postadaptation period. The aftereffect in stride length was retained longer in the medium-resistance load condition $(\sim 18 \% \pm 5 \%$ of MVC) than in the heavy- $(\sim 24 \% \pm$ $6 \%$ of MVC) and light-resistance $(\sim 12 \% \pm 3 \%$ of MVC) load conditions. These results suggest that the kinematic error size that subjects experience during the adaptation period may modulate the retention of the aftereffect in stride length during the postadaptation period. Thus, in order to prolong the retention of the aftereffect in stride length during locomotor training in human SCI, the error size needs to be optimized.

We observed an aftereffect consisting of an increase in stride length during the postadaptation period following release of the resistance load, which was consistent with previous studies on patients with incomplete SCI [15-16]. In particular, the aftereffect was retained relatively longer in our study (i.e., 11 strides for the light-, 17 strides for the medium-, and 14 strides for the heavyresistance load conditions) than in a previous study that used the Lokomat to generate swing-phase resistance 

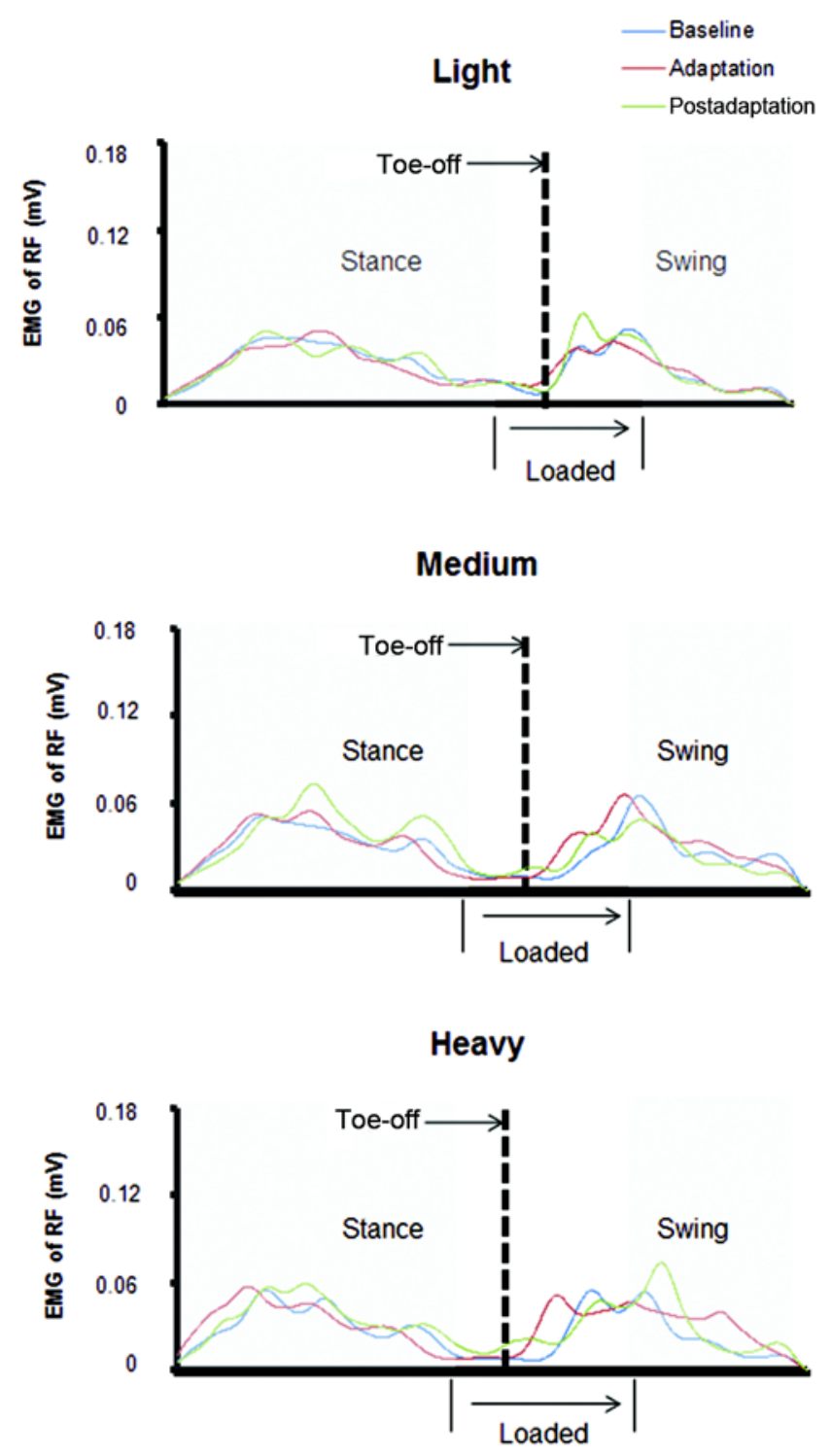

Figure 8.

Subject's rectus femoris (RF) electromyography (EMG) pattern during baseline, adaptation period, and postadaptation period in each resistance load condition. Vertical dashed line represents event of toe-off of right leg, which separates stance and swing phases.

[15]. Specifically, the earlier study compared the stride lengths recorded during baseline with those recorded at strides 1, 10, and 20 following removal of resistance load. Although an increase in stride length was observed after resistance load removal, the increase only reached statistical significance at stride 1 . The difference in the retention interval observed in this and previous study

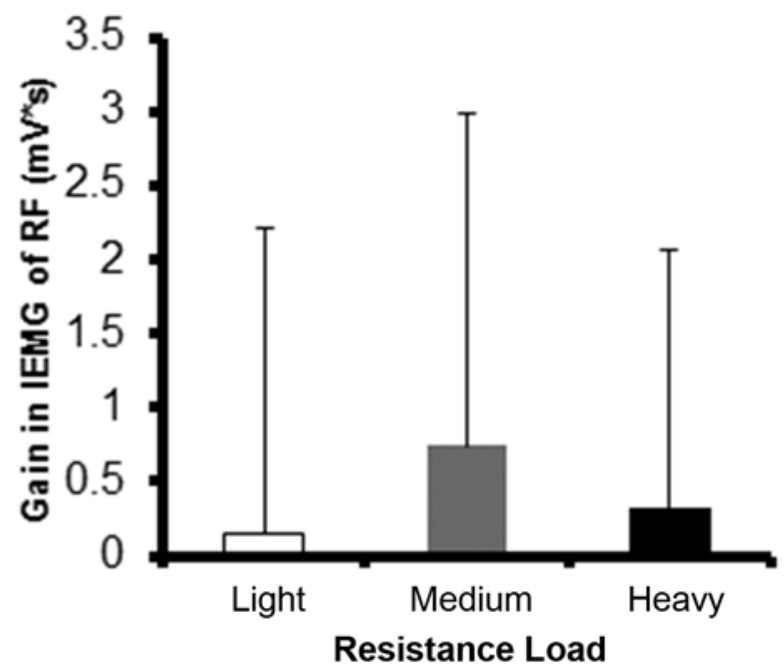

Figure 9.

Group's mean gain/loss in integrated electromyography (IEMG) of rectus femoris (RF) during first 10 strides of adaptation period in each condition. Error bars represent standard deviation.

could be due to the difference in methodology. However, this difference could also be because our test apparatus (i.e., the cable-driven robotic system) was highly backdrivable [17] and had less friction and inertia than the Lokomat, which used the ball-screw mechanism [19].

The adaptation process to large and small errors is likely different on a neural level. For instance, the cerebellum may be involved in the adaptation process involving large errors, but not with small errors, as evident by the finding that cerebellar degeneration compromised the former to a greater extent than the latter [7]. In addition, the prefrontal cortical regions may be involved in adaptation to large errors that produce conscious awareness of the error, but not involved in adaptation to small errors that do not produce such awareness [20]. Along this line of thinking, we speculated that the motor memory produced by different error sizes may be stored in different regions of the brain, which may be a cause for the various levels of retention of the aftereffect.

The spinal cord plays an important role in conveying errors during locomotor adaptation. Specifically, error signals generated by the resistance load reach the brain through the proprioceptive ascending pathway [21]. Damages to the spinal cord could block the error signal and in turn affect the adaptation process. For example, the relatively short retention time observed in the lightresistance load condition may be caused by (1) small 
error size (Figure 2(a)) and (2) further reduction in the error signal during transmission through the impaired proprioceptive pathway. In consideration of this, it is feasible that a weak (e.g., small) error signal may not be strong enough to induce changes at a neural level (e.g., adaptation) in humans with SCI, resulting in an unstable aftereffect.

The aftereffect of an increased stride length was retained for a shorter period of time in the heavy-resistance load condition than in the medium-resistance load condition, suggesting that a large error size may undermine the retention of aftereffects. Fatigue was another factor that may contribute to this shorter retention time. The heavyresistance load condition was 30 percent greater than the medium-resistance load condition, which may overwork the leg muscles and have a negative effect on subjects' walking performance during the postadaptation period. In this particular study, fatigue may not be the only reason for a shorter retention time of the aftereffect observed in the heavy-resistance load condition, because the retention of the aftereffect was also shorter in the light-resistance load condition (when compared with the medium-resistance load condition). Another possible explanation for the shorter retention time in the heavy-resistance load condition was that cognitive strategies may be employed to quickly reduce errors in response to a large perturbation, but this rapid performance improvement also vanishes quickly after that perturbation is removed, leading to a faster decline of aftereffects during the postadaptation period [22]. In addition, it was suggested that two distinct computational processes may be involved during motor adaptation with faster learning process, which is often induced by larger errors and often retains for a shorter period of time in comparison with the condition with slower learning process [23].

Our subjects with incomplete SCI produced an aftereffect in stride length but not in swing time following adaptation to resistance. This suggests that the control mechanisms for spatial and temporal gait parameters in humans with incomplete SCI may be different in locomotor adaptation. This point is supported by previous studies in both the patient population and among nondisabled controls. For example, children with a hemispherectomy have difficulty in adapting temporal parameters but not spatial parameters [24]. Nondisabled children develop the ability to adapt temporal parameters earlier than they do to spatial parameters [25]. Distraction affects the adaptation rate of spatial parameters but not that of tem- poral parameters in nondisabled adults [26], suggesting different neural pathways may be involved in the control of spatial and temporal parameters during walking. In particular, previous evidence suggest that the cerebellum is a critical structure involved in the locomotor adaptation [27], although it may not be directly affected by the spinal cord lesion. The different control mechanisms for spatial and temporal parameters should be considered carefully when locomotor adaptation is applied to clinical setting to improve locomotor function in humans with SCI. It is important to consider that the type of adaptation training chosen could improve one type of gait parameter but not the other.

Locomotor adaptation to swing-phase resistance has been shown to increase stride length in patients with incomplete SCI [15-16], and therefore has the potential to be used clinically to facilitate gait recovery. This current study suggests that the magnitude of the resistance is an important parameter to be controlled in this type of training paradigm, and that there may be an optimal level of resistance that is patient-specific that will induce the greatest retention in the aftereffect. Specifically, a heavyresistance load resulting in large errors and a lightresistance load resulting in small errors may both be suboptimal to maximize the retention of the aftereffect in patients with SCI.

It is important to note that we did not expect a large difference in the retention level between the test conditions (e.g., light-, medium-, and heavy-resistance loads) following only one session of adaptation to resistance. To have a clinically significant change in gait behavior, long-term repeated adaptations would be needed to accumulate the aftereffects over time [1]. In order to maximize long-term motor adaptation training effect, it is important to facilitate the highest level of retention of aftereffects within each training session. This can be achieved through the use of a level of resistance that will result in the highest retention of aftereffects.

We were not able to identify the muscles that drove the increase in stride length based on our EMG data. Our subjects with incomplete SCI did not show a significant increase in the RF activity from baseline when the load was applied. This finding was consistent with a previous study in which subjects with incomplete SCI did not show a significant increase in the RF activity when leg weights or a resistance force generated by the Lokomat were applied to the leg during gait [28]. We speculated that patients with incomplete SCI may recruit the iliopsoas, 
which is another major hip flexor, to counterbalance the resistance load. However, the iliopsoas is difficult to access with surface EMG and, therefore, was not measured in our study. On the other hand, we found that the subjects' TA activity tended to increase as the resistance load increased, which was also consistent with the previous study [28]. The increased TA muscle activity may suggest that the voluntary descending drive was increased due to the resistance load, and the strength of the voluntary drive increased as the resistance load increased. Overall, further study using more sophisticated EMG measuring techniques is warranted to test our speculation regarding RF activity and promote a greater understanding of the muscle activity driving the changes seen in stride length in response to a resistance load during adaptation training.

A limitation of this study was the variation in subjects' levels and severity of SCI. Subjects' SCI levels ranged from $\mathrm{C} 1$ to $\mathrm{T} 7$ and their self-selected walking speeds (as a measure of function) ranged from 0.50 to $0.82 \mathrm{~m} / \mathrm{s}$. These factors may have contributed to the relatively large variability in the subjects' responses to the resistance load. Also, because of the small sample size, we are not able to make a conclusion about the effect of SCI level and severity on the retention of the aftereffect in patients with incomplete SCI. In addition, two subjects required body-weight support in order to complete the adaptation tasks (subjects 1 and 3,20\% of their body weight, Table 2). While these subjects' data were generally consistent with the group trend (e.g., retention of aftereffect was longer in the medium-resistance load condition), the effect of body-weight support on the relationship between the error size and retention of aftereffects should be investigated more rigorously in a future study with more subjects. Furthermore, error size was calculated by taking the difference between the adaptation and baseline values, with the baseline value calculated by taking the mean value across the last 20 strides of the baseline period. While this approach is convenient and has been used by other investigators in the field [7], the results should be cautiously interpreted because it actually eliminates the variability inherent in the baseline period.

In this study, we were not able to determine whether and how the mechanical constraint from the treadmill affected the retention of the aftereffect. Subjects could have voluntarily reduced their stride length in the postadaptation period in order to walk at a pace that was consistent with the moving treadmill belt, given that the belt speed was constant. Future study may investigate the retention of the aftereffect in an overground context following locomotor adaptation on a treadmill. Another limitation was that subjects experienced varying levels of resistance load in the process of determining the comfortable resistance load prior to data collection. Thus, the resistance load was not a novel perturbation for the subjects during the test, which may potentially affect the results. Lastly, future study should investigate the difference between unilateral and bilateral force perturbations in regards to adaptation and retention of the aftereffect. In this study, we investigated only unilateral force perturbations. Patients with incomplete SCI usually demonstrate abnormalities in both legs during gait. Thus, understanding how they adapt their gait pattern in response to a bilateral force perturbation may further the knowledge of motor adaptation on gait recovery in humans with SCI.

\section{CONCLUSIONS}

In this study, we applied different levels of swingphase resistance load to the leg during treadmill walking to investigate the relationship between error size and retention of the aftereffect in patients with incomplete SCI. We found that following short-term adaptation, the patients retained the aftereffect of an increased stride length for a longer period when the error size was intermediate (i.e, medium-resistance load condition). The results suggest that an optimal amount of error in leg kinematics may be needed to enhance the retention of locomotor adaptation. Results from this study may provide suggestions in the development of patient- and taskspecific therapeutic interventions to improve locomotor function in humans with SCI.

\section{ACKNOWLEDGMENTS}

\section{Author Contributions:}

Study concept and design: S. Yen, M. Wu.

Acquisition of data: S. Yen, J. M. Landry, M. Wu.

Analysis and interpretation of data: S. Yen, M. Wu.

Drafting of manuscript: S. Yen.

Critical revision of manuscript for important intellectual content: M. Wu.

Statistical analysis: S. Yen.

Obtained funding: $\mathrm{M}$. Wu.

Administrative, technical, or material support: S. Yen, J. M. Landry, M. Wu. 
Study supervision: M. Wu.

Financial Disclosures: The authors have declared that no competing interests exist.

Funding/Support: This material was based on work supported by the Craig H. Neilsen Foundation (grant 124890).

Additional Contributions: We thank Drs. Brian D. Schmit and T. George Hornby for their thoughtful suggestions for this study. Institutional Review: Informed consent was obtained and all procedures were conducted in accordance with the Helsinki Declaration of 1975 and approved by the Institutional Review Board of Northwestern University.

Participant Follow-Up: The authors do not plan to inform participants of the publication of this study. However, participants have been encouraged to check the study Web site for updated publications.

\section{REFERENCES}

1. Bastian AJ. Understanding sensorimotor adaptation and learning for rehabilitation. Curr Opin Neurol. 2008;21(6): 628-33. [PMID:18989103] http://dx.doi.org/10.1097/WCO.0b013e328315a293

2. Noble JW, Prentice SD. Adaptation to unilateral change in lower limb mechanical properties during human walking. Exp Brain Res. 2006;169(4):482-95. [PMID:16328304] http://dx.doi.org/10.1007/s00221-005-0162-3

3. Emken JL, Reinkensmeyer DJ. Robot-enhanced motor learning: Accelerating internal model formation during locomotion by transient dynamic amplification. IEEE Trans Neural Syst Rehabil Eng. 2005;13(1):33-39. [PMID:15813404] http://dx.doi.org/10.1109/TNSRE.2004.843173

4. Blanchette A, Bouyer LJ. Timing-specific transfer of adapted muscle activity after walking in an elastic force field. J Neurophysiol. 2009;102(1):568-77.

[PMID:19420121] http://dx.doi.org/10.1152/jn.91096.2008

5. Lam T, Anderschitz M, Dietz V. Contribution of feedback and feedforward strategies to locomotor adaptations. J Neurophysiol. 2006;95(2):766-73. [PMID:16424453] http://dx.doi.org/10.1152/jn.00473.2005

6. Huang VS, Shadmehr R. Persistence of motor memories reflects statistics of the learning event. J Neurophysiol. 2009; 102(2):931-40. [PMID:19494195] http://dx.doi.org/10.1152/jn.00237.2009

7. Criscimagna-Hemminger SE, Bastian AJ, Shadmehr R. Size of error affects cerebellar contributions to motor learning. J Neurophysiol. 2010;103(4):2275-84. [PMID:20164398] http://dx.doi.org/10.1152/jn.00822.2009

8. Hatada Y, Rossetti Y, Miall RC. Long-lasting aftereffect of a single prism adaptation: Shifts in vision and proprioception are independent. Exp Brain Res. 2006;173(3):415-24.
[PMID:16552560]

http://dx.doi.org/10.1007/s00221-006-0381-2

9. Klassen J, Tong C, Flanagan JR. Learning and recall of incremental kinematic and dynamic sensorimotor transformations. Exp Brain Res. 2005;164(2):250-59.

[PMID:15947919]

http://dx.doi.org/10.1007/s00221-005-2247-4

10. Imamizu H, Miyauchi S, Tamada T, Sasaki Y, Takino R, Pütz B, Yoshioka T, Kawato M. Human cerebellar activity reflecting an acquired internal model of a new tool. Nature. 2000;403(6766):192-95. [PMID:10646603] http://dx.doi.org/10.1038/35003194

11. Ohyama T, Medina JF, Nores WL, Mauk MD. Trying to understand the cerebellum well enough to build one. Ann N Y Acad Sci. 2002;978:425-38. [PMID:12582071] http://dx.doi.org/10.1111/j.1749-6632.2002.tb07585.x

12. Morton SM, Bastian AJ. Cerebellar contributions to locomotor adaptations during splitbelt treadmill walking. J Neurosci. 2006;26(36):9107-16. [PMID:16957067] http://dx.doi.org/10.1523/JNEUROSCI.2622-06.2006

13. Duysens J, Van de Crommert HW. Neural control of locomotion; The central pattern generator from cats to humans. Gait Posture. 1998;7(2):131-41. [PMID:10200383] http://dx.doi.org/10.1016/S0966-6362(97)00042-8

14. Krawetz P, Nance P. Gait analysis of spinal cord injured subjects: Effects of injury level and spasticity. Arch Phys Med Rehabil. 1996;77(7):635-38. [PMID:8669987] http://dx.doi.org/10.1016/S0003-9993(96)90000-3

15. Houldin A, Luttin K, Lam T. Locomotor adaptations and aftereffects to resistance during walking in individuals with spinal cord injury. J Neurophysiol. 2011;106(1):247-58.

[PMID:21543755] http://dx.doi.org/10.1152/jn.00753.2010

16. Yen SC, Schmit BD, Landry JM, Roth H, Wu M. Locomotor adaptation to resistance during treadmill training transfers to overground walking in human SCI. Exp Brain Res. 2012;216(3):473-82. [PMID:22108702] http://dx.doi.org/10.1007/s00221-011-2950-2

17. Wu M, Hornby TG, Landry JM, Roth H, Schmit BD. A cable-driven locomotor training system for restoration of gait in human SCI. Gait Posture. 2011;33(2):256-60. [PMID:21232961] http://dx.doi.org/10.1016/j.gaitpost.2010.11.016

18. Zeni JA Jr, Richards JG, Higginson JS. Two simple methods for determining gait events during treadmill and overground walking using kinematic data. Gait Posture. 2008; 27(4):710-14. [PMID:17723303] http://dx.doi.org/10.1016/j.gaitpost.2007.07.007

19. Colombo G, Joerg M, Schreier R, Dietz V. Treadmill training of paraplegic patients using a robotic orthosis. J Rehabil Res Dev. 2000;37(6):693-700. [PMID:11321005] 
20. Shadmehr R, Holcomb HH. Neural correlates of motor memory consolidation. Science. 1997;277(5327):821-25. [PMID:9242612] http://dx.doi.org/10.1126/science.277.5327.821

21. Lam T, Pearson KG. Proprioceptive modulation of hip flexor activity during the swing phase of locomotion in decerebrate cats. J Neurophysiol. 2001;86(3):1321-32. [PMID:11535680]

22. Wong AL, Shelhamer M. Saccade adaptation improves in response to a gradually introduced stimulus perturbation. Neurosci Lett. 2011;500(3):207-11. [PMID:21741440] http://dx.doi.org/10.1016/j.neulet.2011.06.039

23. Smith MA, Ghazizadeh A, Shadmehr R. Interacting adaptive processes with different timescales underlie short-term motor learning. PLoS Biol. 2006;4(6):e179.

[PMID:16700627]

http://dx.doi.org/10.1371/journal.pbio.0040179

24. Choi JT, Vining EP, Reisman DS, Bastian AJ. Walking flexibility after hemispherectomy: Split-belt treadmill adaptation and feedback control. Brain. 2009;132(Pt 3):722-33. [PMID:19074191] http://dx.doi.org/10.1093/brain/awn333

25. Vasudevan EV, Torres-Oviedo G, Morton SM, Yang JF, Bastian AJ. Younger is not always better: Development of locomotor adaptation from childhood to adulthood. J Neurosci. 2011;31(8):3055-65. [PMID:21414926] http://dx.doi.org/10.1523/JNEUROSCI.5781-10.2011

26. Malone LA, Bastian AJ. Thinking about walking: Effects of conscious correction versus distraction on locomotor adaptation. J Neurophysiol. 2010;103(4):1954-62.

[PMID:20147417]

http://dx.doi.org/10.1152/jn.00832.2009

27. Reisman DS, Bastian AJ, Morton SM. Neurophysiologic and rehabilitation insights from the split-belt and other locomotor adaptation paradigms. Phys Ther. 2010;90(2): 187-95. [PMID:20023001] http://dx.doi.org/10.2522/ptj.20090073

28. Lam T, Wirz M, Lünenburger L, Dietz V. Swing phase resistance enhances flexor muscle activity during treadmill locomotion in incomplete spinal cord injury. Neurorehabil Neural Repair. 2008;22(5):438-46. [PMID:18780879] http://dx.doi.org/10.1177/1545968308315595

Submitted for publication September 25, 2012. Accepted in revised form April 1, 2013.

This article and any supplementary material should be cited as follows:

Yen S, Landry JM, Wu M. Size of kinematic error affects retention of locomotor adaptation in human spinal cord injury. J Rehabil Res Dev. 2013;50(9):1187-1200. http://dx.doi.org/10.1682/JRRD.2012.09.0175

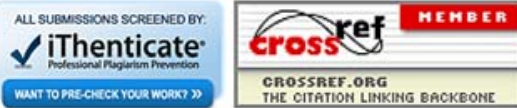

